\title{
Match-Play Running Demands and Technical Performance Among Elite Gaelic Footballers: Does Divisional Status Count?
}

\author{
Jason Mcgahan \\ Cork Institute of Technology \\ Shane Mangan \\ Technological University Dublin, shane.mangan@tudublin.ie \\ Kieran Collins \\ Technological University Dublin, kieran.collins@tudublin.ie
}

See next page for additional authors

Follow this and additional works at: https://arrow.tudublin.ie/ittsciart

Part of the Sports Sciences Commons

\section{Recommended Citation}

McGahan, J., Mangan, S. \& Collins, K. (2021). Match-Play Running Demands and Technical Performance Among Elite Gaelic Footballers: Does Divisional Status Count? Journal of Strength and Conditioning Research, vol. 35, no. 1, pg. 169-175. doi:10.1519/JSC.0000000000002450

This Article is brought to you for free and open access by the School of Science and Computing at ARROW@TU Dublin. It has been accepted for inclusion in Articles by an authorized administrator of ARROW@TU Dublin. For more information, please contact arrow.admin@tudublin.ie, aisling.coyne@tudublin.ie, gerard.connolly@tudublin.ie.

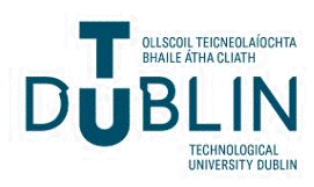




\section{Authors}

Jason Mcgahan, Shane Mangan, Kieran Collins, and Con Byrnes

This article is available at ARROW@TU Dublin: https://arrow.tudublin.ie/ittsciart/126 
See discussions, stats, and author profiles for this publication at: https://www.researchgate.net/publication/322727546

\section{Match-Play Running Demands and Technical Performance Among Elite Gaelic Footballers: Does Divisional Status Count?}

Article in The Journal of Strength and Conditioning Research · January 2018

DOI: 10.1519/JSC.0000000000002450

\section{CITATIONS}

18

6 authors, including:

Jason Mcgahan

Cork Institute of Technology

18 PUBLICATIONS 122 CITATIONS

SEE PROFILE

Kieran Collins

Technological University Dublin - Tallaght Campus

132 PUBLICATIONS 1,939 CITATIONS

SEE PROFILE

Some of the authors of this publication are also working on these related projects:

Project Project Spraoi View project

Project Project Spraoi View project
514

Shane Mangan

Technological University Dublin - Tallaght Campus

28 PUBLICATIONS 150 CITATIONS

SEE PROFILE

Con Burns

Cork Institute of Technology

59 PUBLICATIONS 7,875 CITATIONS

SEE PROFILE 
Running Title: Physical \& technical measures in Gaelic football

Article Title: Match-play running demands and technical performance among elite Gaelic footballers: Does divisional status count?

Authors: Jason H McGahan. ${ }^{1}$ Shane Mangan. ${ }^{2}$ Kieran Collins ${ }^{2}$ Con Burns. ${ }^{1}$ Tim Gabbett. ${ }^{3,4}$ Cian O’ Neill. ${ }^{1}$

Affiliations: ${ }^{1}$ Department of Sport, Leisure \& Childhood Studies, Cork Institute of Technology. ${ }^{2}$ Gaelic Sports Research Centre, Institute of Technology Tallaght. ${ }^{3}$ Gabbett Performance Solutions, Australia. 4 Institute for Resilient Regions, University of Southern Queensland, Australia

Correspondence to:

Mr Jason McGahan

Department of Sport, Leisure \& Childhood Studies, Cork Institute of Technology, Rossa Avenue, Bishopstown, County Cork, Ireland

Telephone: 0214326142

Email: jason.mcgahan@mycit.ie

Submission Type: Original Investigation

Abstract Word Count: 274

Text-Only Word Count: 2995

Number of Figures and Tables: 4 tables 


\section{Abstract}

2 The aim of the current study was to compare positional differences in running demands and 3 technical performance variables among elite Gaelic football teams from separate Divisions.

4 Data were obtained from a Division $1(26.7 \pm 2.9$ years, $179.2 \pm 21.3 \mathrm{~cm}, 89.9 \pm 21.2 \mathrm{~kg})$ and

5 a Division $3(25.7 \pm 3.5$ years, $183.0 \pm 4.7 \mathrm{~cm}, 84.4 \pm 6.5 \mathrm{~kg})$ team. Match-play running

6 variables were collected using 4-Hz global positioning system (GPS) units (VX Sport, New-

7 Zealand) (Match data sets; Division 1: $n=107$, Division 3: $n=97$ ). Selected variables

8 assessed were high speed running distance (HSR) $\left(\geq 17 \mathrm{~km} \cdot \mathrm{h}^{-1}\right)$, number of high-speed efforts

$9(\mathrm{HSE})\left(\geq 17 \mathrm{~km} \cdot \mathrm{h}^{-1}\right)$, relative high-speed distance $(\mathrm{RHSD})\left(\geq 17 \mathrm{~km} \cdot \mathrm{h}^{-1} ; \mathrm{m} \cdot \mathrm{min}^{-1}\right)$ and 10 percentage of time at high speed (\%HS). Each variable was analysed across the 5 positional groups in Gaelic football (full-back, half-back, midfield, half-forward, full-forward). The same 25 competitive games were analysed using GPS and the Sports Code video analysis system (Sports Code Elite V9, Sportstec, NSW, Australia). Technical performance variables selected for analysis were total kick/hand passes, tackles, shots and percentage of time in possession. HSR running demands were differentiated between the divisions; the Division 3 team demonstrated significantly greater HSR, HSE, RHSD and \%HS than the Division 1 team $(p<0.05)$. Positional-specific analysis found that the Division 3 fullback and midfield positional lines had significantly greater HSR, RHSD and \%HS than their Division 1 counterparts. The Division 1 team made a greater number of total tackles, with significantly more tackles in the middle third $(p<0.05)$. The Division 3 team performed a significantly greater number of hand passes and unsuccessful shots per game $(p<0.01)$. The results of the present study indicate that overall technical proficiency, rather than high-speed running profiles, differentiate Division 1 and 3 Gaelic football teams.

Key words: GPS, Divisional-differences, Gaelic games, Video-analysis 


\section{INTRODUCTION}

Gaelic football is an amateur field-based sport and is the most popular sport in Ireland. $(2,9,12)$ The best Gaelic footballers in each of the 32 counties in Ireland are selected to represent their county team who compete in the All Ireland Championship and the National League competitions.(2) The All Ireland Championship competition, the most prestigious competition in Gaelic football, is played by all county teams in a knock out cup format. In the National Football League competition, the second most prestigious competition, the teams are divided into four rank ordered divisions with 8 teams in each division.(26) Teams have the possibility of being promoted or relegated from their respective divisions based on their performance in the National League. In the high performance Gaelic football inter-county environment, considerable time is devoted to the development of the physical running demands amongst other technical and tactical performance-related variables.(24)

The running demands of elite Gaelic football players have been examined for over twenty five years, with an initial emphasis on video and time-motion analysis. $(17,29)$ Given the advancement in technology, global positioning systems (GPS) have been the tool of choice to investigate the running demands of the modern Gaelic football player. $(6,24,26$, 31) Early research highlighted differences between the 5 positional lines (i.e. full back, half back, mid-field, half forward, full forward) with regard to the respective running demands during match-play. (24) The study found significant differences among the middle 8 players (half-backs, midfielders and half-forwards) covering greater distances $(8700-9523 \mathrm{~m})$ than the inside line players (full-back 6892m and full-forwards 7090m respectively). However, it should be noted that this study only investigated the running demands of one team within one division of the National Football League (NFL). A more recent paper by Mangan and colleagues (27) examined changes in running demands across a playing season using multiple teams. 
It was found that teams who progressed to the latter part of the All Ireland

Championship were found to increase their running demands, with these running demands

52

closely related to the score-line of the match (27).

Despite the growth of research investigating the running demands in elite Gaelic football, presently there is a lack of published literature across technical performance variables. More recently Carroll (4) investigated specific technical elements of performance from teams of different standards, concluding that differences in technical performance between teams vary as a result of opposition characteristics. The study also found that attack efficiency and total number of shots were higher for the higher ranked teams when compared to lower ranked teams. Similar findings have been reported within soccer $(21,30)$ and Australian Rules Football (AF) (11) where total shots, shots on target, passes and number of tackles separate the top and bottom ranked teams.

Currently within Gaelic football there is dearth of research comparing the high-speed running demands across standards of play. (26) Although numerous studies have examined the positional demands, $(24,31)$ none have differentiated teams by success rates or divisional status. Studies in AF, $(1,3)$ rugby league (10) and soccer (28) have reported that elite players record greater running demands when compared to their counterparts playing at the sub-elite level. However, in contrast to these studies, it has been reported that soccer and rugby league teams competing in higher divisions of competition cover less total distance and less highspeed running distance than teams competing in lower divisions. $(8,13)$ It has been hypothesized that this is due to their increased technical abilities. Furthermore, Di Salvo et al. (7) found that the bottom 5 teams in the English Premier League covered greater high-speed running distance than the teams in the top 5 positions. However, it is unclear whether similar patterns exist within elite Gaelic football match-play. 
To date, there is no published literature that has investigated the differences in high-

speed running and technical performance variables between different divisional teams in elite

Gaelic football. Analysis of such differences would serve to assist coaches in identifying the key characteristics of performance and could help to increase prospects of promotion to higher divisions. Therefore, the purpose of the current study was to investigate the matchplay high-speed running demands and technical performance variables of Division 1 and Division 3 players in elite Gaelic football.

\section{METHODS}

\section{Experimental Approach to the Problem}

Data were collected across a full Division 1 and Division 3 season (9 months, JanSept) which consisted of 25 competitive games in total (Division 1: $n=13$; Division 3: $n=$ 12). Data included League games (Division 1: $n=7$; Division 3: $n=8$ ) and Championship games (Division 1: $n=6$; Division 3: $n=4$ ). Post-season ranking placed both teams in the top 3 of their respective divisions. Only full individual match data sets (i.e. players who completed the full match 75-80min) were selected for analysis (Division 1: $n=107$; Division 3: $n=97)$. Notably, an initial analysis revealed no significant difference between the running and technical demands of each team across their respective league and championship competitions, therefore all matches, irrespective of phase of season, were analysed together. Players were sub-categorized via the five positional lines in Gaelic football, full-back (Division 1: $n=29$; Division 3: $n=23$ ), half-back (Division 1: $n=30$; Division 3: $n=22$ ), midfield (Division 1: $n=13$; Division 3: $n=19$ ), half-forward (Division 1: $n=15$; Division 3: $n=16$ ) and full-forward (Division 1: $n=20$; Division 3: $n=17$ ). 
97

98

99

100

101

102

103

104

105

106

107

108

109

110

111

112

113

114

\section{Subjects}

Forty seven Gaelic football players from two elite teams volunteered to participate in the study; 23 from a Division 1 team $(26.7 \pm 2.9$ years, $179.2 \pm 21.3 \mathrm{~cm}, 89.9 \pm 21.2 \mathrm{~kg})$ and 24 from a Division 3 team $(25.7 \pm 3.5$ years, $183.0 \pm 4.7 \mathrm{~cm}, 84.4 \pm 6.5 \mathrm{~kg})$. This study received ethical approval from the local institution's Human Research Ethics Committee. Players were provided with a detailed explanation and completed a consent form prior to commencement of data collection.

\section{Procedure}

Running Demands

The running-based movement demands were assessed using VX Sport 4 Hz GPS units (VX Sport; Visuallex Sport, Lower Hutt, New Zealand, Firmware: V1.60 28). The unit was worn on the upper back between the shoulder blades; players wore the same unit across all competitive matches. All devices were activated by the side of the pitch, satellite locked and established for a minimum of 30 minutes before the commencement of each match. $(22,24)$ Following each match, running performance data was downloaded using the VX software suite (VX Sport View, New Zealand V1.60 28). Each file was trimmed to ensure that only data recorded when the player was in competition (excluding warm-up, half-time and cooldown) was included for analysis.

The VX Sport GPS unit has been found to be a valid and reliable tool for the measurement of intermittent activity in field sports.(23) The typical error (TE $\pm 95 \%$ confidence interval $[\mathrm{CI}]$ ) was $0.75 \pm 0.26$ for maximum speed, and $0.55 \pm 0.19$ for average speed (23). The coefficient of variation $(\mathrm{CV} \% \pm 95 \% \mathrm{CI})$ was $4.2 \pm 1.5$ for maximum speed, and $4.4 \pm 1.5$ for average speed.(23) The running variables assessed were high speed running distance (HSR) $\left(\geq 17 \mathrm{~km} \cdot \mathrm{h}^{-1}\right)$, number of speed efforts (HSE) $\left(\geq 17 \mathrm{~km} \cdot \mathrm{h}^{-1}\right)$, relative high 
121 speed distance (RHSD) per $\min \left(\mathrm{m} \cdot \mathrm{min}^{-1}\right)$ and percentage of time spent at high speed $(\%$ 122 HS). Acceleration were defined as a change in speed by $2 \mathrm{~km} \cdot \mathrm{h}^{-1}$ within 1 second. The

123

124 change was triggered over a minimum time of 2 seconds. The acceleration stopped when the player decelerated to $<75 \%$ of maximum speed reached in the forgoing sprint effort.(24) These variables were analyzed for all 5 out-field positional groups. Selected thresholds and metrics used in the current study have been used previously in the GAA literature. $(6,24,25)$

Video Analysis

Sports code video analysis (Sports Code Elite V9, Sportstec, Warriewood, New South Wales, Australia) was used to determine the technical performance of Gaelic football matchplay on an iMac (version OS X 10.9.4). A specific coding template was developed to best understand the technical variables within match-play. Each technical variable analyzed was assigned an operational definition to determine what was deemed to be a successful or unsuccessful outcome (Table 1). For the purpose of this research, the Gaelic football pitch was divided into 3 distinct sections (Table 1). Two games were chosen at random to perform test-retest reliability on the coding of technical actions. This involved re-coding the 2 games 7-10 days after the original coding. Test-retest reliability was conducted for each of the variables using paired sample t-tests. There was a very low percentage error for all variables $(<2 \%)$

\section{Insert Table 1 About Here}

\section{Statistical Analysis}

All data are reported as mean \pm standard deviation unless stated. Preliminary assumption testing was conducted to check for normality (Shapiro Wilk test), while Levene's test was used to test the homogeneity of variances. Independent sample $t$-tests were used to compare the mean performance scores (independent of position) of both teams for all GPS 
and technical performance variables. The statistical significance was set at $p<0.05$. Further position-specific independent sample $t$-tests were used to compare GPS variables between players in the same positional lines on both teams. Eta squared effect size (95\% CI) was used to determine the magnitude of any statistical difference (Trivial effect $\eta^{2}<0.01$; Small effect: $0.01 \leq \eta^{2}<0.06$; Medium effect: $0.06 \leq \eta^{2}<0.14$; Large effect: $\eta^{2} \geq 0.14$ ). (5) All data was analyzed using IBM SPSS (Statistical Package for Social Studies), Version 22.

\section{RESULTS}

There were significant differences across all the GPS variables HSR $\left(p=.001, \eta^{2}=\right.$ $.054, \mathrm{ES}=$ small $), \operatorname{HSE}\left(p=.024, \eta^{2}=.025, \mathrm{ES}=\operatorname{small}\right), \operatorname{RHSD}\left(p=.002, \eta^{2}=.047, \mathrm{ES}=\right.$ small $)$ and $\%$ HS $\left(p=.002, \eta^{2}=.049, \mathrm{ES}=\right.$ small $)$ with the Division 3 team consistently demonstrating significantly higher scores than their Division 1 counterparts (Table 2).

\section{Insert Table 2 About Here}

Position-specific analyses (5 positional lines) comparing running demands between Division 1 and Division 3 players is illustrated in Table 2. The Division 3 full-backs had higher HSR $\left(p=.002, \eta^{2}=.177, \mathrm{ES}=\right.$ large $), \operatorname{RHSD}\left(p=.002, \eta^{2}=.174, \mathrm{ES}=\right.$ large $)$ and $\%$ HS $\left(p=.001, \eta^{2}=.208, \mathrm{ES}=\right.$ large $)$ than their Division 1 counterparts. Division 3 midfielders also performed significantly more HSR $\left(p=.001, \eta^{2}=.520, \mathrm{ES}=\right.$ large $), \operatorname{RHSD}\left(p=.001, \eta^{2}=\right.$ $.508, \mathrm{ES}=$ large $), \% \mathrm{HS}\left(p=.001, \eta^{2}=.431, \mathrm{ES}=\right.$ large $)$ and additionally more HSE $(p<0.01$, $\eta^{2}=0.301 ; \mathrm{ES}=$ large) than their Division 1 counterparts (Figure 1). There were no significant differences between half-backs, half-forwards and full-forwards for any of the GPS variables. 

in Table 3. The Division 3 team demonstrated a significantly greater number of total hand passes $\left(p=.007, \eta^{2}=.278, \mathrm{ES}=\right.$ large $)$ and missed shots per game $\left(p=.007, \eta^{2}=.279, \mathrm{ES}=\right.$

171 large) than the Division 1 team. There were no significant differences between the teams for total kick passes $\left(p=.102, \eta^{2}=.112, \mathrm{ES}=\right.$ medium $)$, shots scored from play $\left(p=.606, \eta^{2}=\right.$ $.012, \mathrm{ES}=$ small $)$ and percentage of time in possession $\left(p=.539, \eta^{2}=.017, \mathrm{ES}=\right.$ small $)$. The

Division 1 team made a greater number of total tackles than the Division 3 team, with significantly more tackles in the middle third $\left(p=.044, \eta^{2}=.072\right.$, ES = medium).

\section{DISCUSSION}

The current study is one of the first to investigate the high-speed running demands and technical performance variables in Gaelic football match-play between two elite teams with differing Divisional status. One major finding within this study was that the Division 3 Gaelic football team covered greater HSR, RHSD, performed more HSE and had a higher \% HS running performance when contrasted to the Division 1 team. These differences were most pronounced in the full-back and midfield positional lines. Second unique finding, there were a greater number of tackles, particularly in the middle third, executed by the Division 1 team. Finally, the Division 1 team's attacking efficiency and accuracy in scoring attempts was higher when compared to the Division 3 team, with the latter found to have a greater number of hand passes completed than their Division 1 counterparts. the other two positional lines (full-back and full-forwards). 
These differences in running demands might be explained by the typical interchangeable and roving tactical role the middle 8 players have within Gaelic football (winning possession and transitioning from defense to attack). Additionally, with the previous literature $(24,25)$, the Division 3 midfielders recorded the greatest high speed running values in comparison to the other four positional lines. However in contrast, the findings within the current study, found the Division 1 team's half-forward line covered the greatest high speed running distances. This difference may be due to a tactical ploy among the Division 1 team.

A novel finding within the present study was that the Division 3 full-back and midfield lines had the increased running demands (i.e. HSD, RHSD, HSE and \%HS) when compared to their Division 1 counterparts. There are several possible explanations for this finding. Firstly, the lack of tackles by Division 3 teams in the middle third may result in an increase in match-play involvements for the full-back line, therefore these lines will have increased running demands placed upon them,. This is in direct contrast to the increased tackle count noted within Division 1 teams who had lower running demands but a higher tackle count in the middle third. Notably, the Division 3 team completed a significantly greater number of hand passes; this finding is not surprising as anecdotal evidence suggests that Division 3 teams will engage in a style of play that is hand pass oriented with the aim of keeping possession and working the ball up the pitch (4), which in turn would increase these teams' running demands. In contrast to Division 3 teams, the Division 1 team had a greater number of kick passes than their Division 3 counterparts; this may directly explain the lower high-speed running observed for these players as the ball is consistently traveling a greater distance thus lowering the running demands on these players.

The present findings are in agreement with other research $(7,8,13,26,30)$ that increased high-speed running does not necessarily ensure success. These studies indicate that there are technical and tactical variables at play that more accurately determine success than 
high-speed running. Similar to Hulin et al. (13) within rugby league, the present study found no significant difference between the two teams with regard to the percentage of time in possession of the ball. Previous research has found that the team with less time in possession completes greater amounts of high-speed running defending and chasing $(10,11)$ this was not the case in the present study with both teams having possession of the ball for approximately $50 \%$ of the time.

Another unique finding within the present study was the Division 1 team's execution of a greater number of total tackles. Similar findings were found in rugby league (13) with successful teams making greater number of collisions than unsuccessful teams. A potential explanation for the greater amount of tackles associated with the Division 1 team is the tactical approach selected. Currently, within Gaelic football, there is an increase in teams adopting a high press of kick outs and direct opposition within the half forward and full forward lines with the aim of dispossessing opponent lines (half back and full back) in the middle third. This may have impacted a number of findings within the current study, such as the Division 1 team completing a greater number of tackles in the middle third, resulting in the team winning possession higher up the pitch, which in turn reduced the need for the midfield line to complete high-speed running in their defensive third. Finally if possession is re-gained through this high-press tactic, it will result in the exclusion of the defensive lines from completing high-speed running during the ensuing phases of play.

The Division 1 team demonstrated a higher score rate than the Division 3 team. The 'shots missed' category was greater in the Division 3 team, which may suggest the presence of better decision-making and technical proficiency in front of goal in higher level teams. This finding is consistent with Carroll (4) who found this to be a major difference between higher and lower ranked Gaelic football teams. 
The findings from the current study must be viewed in context with the limitations within the investigation. The use of a $4-\mathrm{Hz}$ GPS system to accurately track high-speed movements must be considered. In addition, the absence of a tri-axial accelerometer and gyroscope, which provides information on physical contact such as a tackle, could have provided additional information related to match-play demands. Future research should aim to apply recent technological advances in this field to evaluate other physiological demands such as contact force (i.e. tackles), change of direction and associated heart rate variability. Furthermore, this study did not examine positional differences in technical performance. Future research should aim to examine the technical performance by position, by team and how these potentially fluctuate across the season. While this study examines differences in movement demands and technical performance variables across divisions, it does not examine differences within teams competing in the same division. Similar to Carrol's (4) findings, a team's style of play and prevalence of technical skill may vary depending on the opponent. Future research in Gaelic football should examine differences in the aforementioned variables from multiple teams within the same division to gather a more overview and representation of performance measures. Finally, future studies in Gaelic football must look at the match to match variation in running performance with a team across multiple divisions taking into account the quality of opposition, styles of play, styles of coaching and the margins that are associated with winning and losing, so we can better understand the contextual factors that impact running and technical demands across divisions. 
Findings from the current research suggest that greater amounts of high-speed running are not necessarily related to team ranking in elite Gaelic football. Scoring accuracy, in addition to a higher frequency of tackles, particularly in the middle third, were characteristics more associated with the higher ranked team. It is beneficial for coaches to be aware of the relevant running and technical performance measures associated with higher divisional status. optimize performance. Currently within Gaelic football, there is an increased emphasis on the physical development of athletes. However these findings highlight the importance of technical competence in differentiating between higher and lower-ranked teams. It is recommended that coaches at all levels place these elements as a central tenet of their coaching philosophy to maximize the development of the key performance skills required during match-play. Finally, this study highlights the importance of technical skill proficiency, rather than running performance capability, in distinguishing between higher and lower ranked teams within elite Gaelic football.

\section{ACKNOWLEDGEMENTS}

No external sources of funding were provided during this research. The authors would like to thank the players who took part in this study and the management and coaches for their support.

\section{REFERENCES}

284 1. Aughey RJ. Widening margin in activity profile between elite and sub-elite Australian football: a case study. J Sci Med Sport 16: 382-386, 2013. 
3. Brewer C, Dawson B, Heasman J, Stewart G, Cormack S. Movement pattern comparisons in elite (AFL) and sub-elite (WAFL) Australian football games using GPS. J Sci Med Sport 13: 618-623, 2010.

291

4. Carroll R. Team Performance Indicators in Gaelic Football and Opposition Effects. Int J Perform Anal Sport 13: 703-715, 2013

5. Cohen JW. Statistical power analysis for behavioral sciences. Hillsdale, NJ: Lawerence Erlbaum Associates, 1988.

6. Collins DK, Solan B, and Doran DA. A preliminary investigation into high-intensity activity during elite Gaelic football. $J$ Sports Therapy 6: 10, 2013

7. Di Salvo V, Gregson W, Atkinson G, Tordoff P, and Drust B. Analysis of high intensity activity in Premier League soccer. Int J Sports Med 30: 205-212, 2009.

8. Di Salvo V, Pigozzi F, González-Haro C, Laughlin MS, De Witt JK. Match performance comparison in top English soccer leagues. Int J Sports Med 34: 526-532, 2013.

9. ESRI. The Social Significance of Sport. Econ Social Res Inst Report: 10-21, 2008.

10. Gabbett T. Influence of the opposing team on the physical demands of elite rugby league match play. J Strength Con Res 27: 1629-1635, 2013.

11. Gabbett TJ, Polly C, Dwyer DB, Kearney S, Corvo A. Influence of field position and phase of play on the physical demands of match-play in professional rugby league forwards. J Sci Med Sport 17: 556-561, 2014.

12. Hassan D. Governance and the Gaelic Athletic Association: time to move beyond the amateur ideal? Soccer \& Society 11: 414-427, 2010.

13. Hulin BT, Gabbett TJ, Kearney S, Corvo A. Physical demands of match play in successful and less-successful elite rugby league teams. Int J Sports Physiol Perform 10: 703-710, 2014. 
14. Johnston R, Gabbett, TJ, Jenkins, DG. Influence of number of contact efforts on running performance during game-based activities. Int J Sports Physiol Perform 10: $740-745,2015$

15. Johnston R, Gabbett, TJ, Seibold, AJ, and Jenkins, DG. Influence of physical contact on pacing strategies during game-based activities. Int J Sports Physiol Perform 9: 811-816, 2014.

16. Johnston RD, Gabbett TJ, Seibold AJ, Jenkins, DG. Influence of physical contact on neuromuscular fatigue and markers of muscle damage following small-sided games. $J$ Sci Med Sport 17: 535-540, 2014.

17. Keane S, Reilly T, and Hughes M. Analysis of workrates in Gaelic Football. Aus J Sci Med Sport 25: 100- 102, 1993.

18. Lago-Peñas C, Lago-Ballesteros, J., Dellal, A., \& Gómez, M. Gamerelated statistics that discriminated winning, drawing and losing teams from the Spanish Soccer League. . J Sports Sci Med 9: 288-293, 2010.

19. Liu H, Gómez, M. A., Lago-Peñas, C., \& Sampaio, J. Match statistics related to winning in the group stage of 2014 Brazil FIFA World Cup. J Sports Sci 33: 1205$1213,2015$.

20. Liu H, Hopkins, W. G., \& Gomez, M.A. Modelling relationships between match events and match outcome in elite football. . Eur J Sport Sci 16: 1-10, 2015.

21. Liu H, Miguel-Angel, G., Gonçalves, B., \& Sampaio, J. Technical performance and match-to-match variation in elite football teams. J Sports Sci 34: 509-518., 2015.

22. Maddison. R. and Ni Mhurchu. C. Global positioning system: A new opportunity in physical activity measurement. Int J Behav Nutr and Phys Act 6: 73, 2009.

23. Malone S, Collins DK, McRobert AP, Morton J, and Doran DA. Accuracy and reliability of VXsport global positioning system in intermittent activity. In: 
Proceedings of the 19th Annual Congress of the European College of Sport Science, Amsterdam, 2-5th July, 2014.

24. Malone S, Solan B, Collins K, and Doran D. The positional match running performance in elite Gaelic football. J Strength Con Res 30: 2292 - 2298, 2015.

25. Malone S, Solan B, Collins K, and Doran D. The metabolic power and energetic demands of Elite Gaelic football match play. J Sports Med Phys fit 55: 543 - 549, 2016.

26. Mangan S, Malone S, Ryan M, McGahan J, O’neill C, Burns C, Warne J, Martin D, and Collins K. The influence of match outcome on running performance in elite Gaelic football. Sci Med Football 1: 272 - 279, 2017.

27. Mangan, S. Ryan, M. Shovlin, A. Mc Gahan, J. Malone, S. O’Neill, C. Burns, C. Collins, K. Seasonal changes in Gaelic football match-play running performance. $J$ Strength Con Res epub ahead of print, 2017.

28. Mohr M, Krustrup P, Bangsbo J. Match performance of high-standard soccer players with special reference to development of fatigue. J Sports Sci 21: 519-528, 2003.

29. O'Donoghue P, Donnelly O, Hughes L, and McManus S. Time-motion analysis of Gaelic games. J Sports Sci 22: 255-256, 2004.

30. Rampinini E, Impellizzeri FM, Castagna C, Coutts AJ, and Wisloff U. Technical performance during soccer matches of the Italian Serie A league: effect of fatigue and competitive level. J Sci Med Sport 12: 227-233, 2009.

31. Reilly B, Akubat I, Lyons M, and Collins DK. Match-play Demands of Elite Youth Gaelic Football Using Global Positioning System Tracking. J Strength Con Res 29: 989 - 996, 2014.

32. Yue ZY, Broich, H., \& Mester, J. Statistical analysis for the soccer matches of the first Bundesliga. Int J Sports Sci Coaching 9: 553-560, 2014. 


\section{LEGEND OF TABLE AND FIGURES}

Table 1. The technical variables quantified and there specific definitions.

Table 2. Position specific mean $( \pm \mathrm{SD})$ high-speed running demands within elite Gaelic football competition with respect to Division 1 and Division 3 players.

Table 3. Mean $( \pm \mathrm{SD})$ technical demands within elite Gaelic football competition with respect to Division 1 and Division 3 teams.

Figure 1. Position specific mean $( \pm \mathrm{SD})$ in high-speed running $(\mathrm{m})$ demands of Division 1 and Division 3 players. 
TABLE 1

Variable

\section{Definition}

Total kick passes

The number of attempts made to transfer the ball by foot to a teammate. Successful and unsuccessful.

Total hand passes

The number of attempts made to transfer the ball by hand to a teammate. Successful and unsuccessful.

Tackle

The number of situations where the defending player made physical contact with an opponent player while contesting the ball.

Shots from play

The number of attempts to score, over or above the cross bar from play. Successful and unsuccessful.

Percentage of possession The overall percentage of time a team had in possession of the ball whilst it was in play.

Gaelic football 3 distinct The defensive section was from the defending team's end-line section to their $45 \mathrm{~m}$ line.

The area between the two $45 \mathrm{~m}$ lines was categorized as the middle section (50-60m).

The area from the opposition's 45m line to the opposition's end-line was categorized as the attacking section. 
TABLE 2

\begin{tabular}{|c|c|c|c|c|}
\hline Variable by Position & Division $1(n=107)$ & Division $3(n=97)$ & Effect Size & Difference $95 \%$ CI \\
\hline \multicolumn{5}{|l|}{ All Positions } \\
\hline $\operatorname{HSR}(\mathrm{m})$ & $1145 \pm 436$ & $1358 \pm 462 \div$ & Small & -336.973 to -89.030 \\
\hline HSE (no.) & $64 \pm 21$ & $71 \pm 24^{*}$ & Small & -13.539 to -.982 \\
\hline RHSD (m/min) & $14.9 \pm 5.7$ & $17.6 \pm 6.1 \div$ & Small & -4.1923 to -.9750 \\
\hline HS (\%) & $11.2 \pm 2.8$ & $12.5 \pm 3.1 \ddagger$ & Small & -2.1340 to -.5103 \\
\hline \multicolumn{5}{|l|}{ Full Back } \\
\hline $\operatorname{HSR}(\mathrm{m})$ & $796 \pm 255$ & $1072 \pm 340^{*}$ & & -451.987 to -106.909 \\
\hline HSE (no.) & $47 \pm 16$ & $55 \pm 14$ & Small & -14.434 to 2.167 \\
\hline RHSD (m/min) & $10.4 \pm 3.3$ & $13.9 \pm 4.5^{*}$ & & -5.6780 to -1.3408 \\
\hline $\operatorname{HS}(\%)$ & $9.2 \pm 1.8$ & $11.9 \pm 3.2 \ddagger$ & Large & -4.2117 to -1.1823 \\
\hline \multicolumn{5}{|l|}{ Half Back } \\
\hline $\operatorname{HSR}(\mathrm{m})$ & $1339 \pm 433$ & $1339 \pm 289$ & Trivial & -201.209 to 201.639 \\
\hline HSE (no.) & $75 \pm 23$ & $73 \pm 17$ & Trivial & -8.898 to 13.976 \\
\hline RHSD (m/min) & $17.5 \pm 5.6$ & $17.3 \pm 3.7$ & Trivial & -2.5434 to 2.9731 \\
\hline $\mathrm{HS}(\%)$ & $12.1 \pm 3.0$ & $12.7 \pm 2.0$ & Trivial & -2.0389 to .9601 \\
\hline \multicolumn{5}{|l|}{ Midfielders } \\
\hline $\operatorname{HSR}(\mathrm{m})$ & $1221 \pm 216$ & $1767 \pm 295 \div$ & Large & -742.122 to -350.599 \\
\hline HSE (no.) & $75 \pm 10$ & $93 \pm 15 \div$ & Large & -27.542 to -7.575 \\
\hline RHSD (m/min) & $15.9 \pm 2.7$ & $22.9 \pm 3.9 \div$ & Large & -9.5415 to -4.4172 \\
\hline $\mathrm{HS}(\%)$ & $10.7 \pm 1.7$ & $13.9 \pm 1.9 \div$ & Large & -4.5626 to -1.8245 \\
\hline \multicolumn{5}{|l|}{ Half Forwards } \\
\hline $\operatorname{HSR}(\mathrm{m})$ & $1500 \pm 548$ & $1659 \pm 254$ & Small & -482.951 to 165.476 \\
\hline HSE (no.) & $75 \pm 21$ & $86 \pm 10$ & Medium & -24.073 to 1.473 \\
\hline $\operatorname{RHSD}(\mathrm{m} / \mathrm{min})$ & $19.6 \pm 7.2$ & $21.4 \pm 3.3$ & Small & -5.9757 to 2.5440 \\
\hline HS (\%) & $13.1 \pm 3.4$ & $14.0 \pm 1.5$ & Small & -2.8838 to 1.0804 \\
\hline \multicolumn{5}{|l|}{ Full Forwards } \\
\hline $\operatorname{HSR}(\mathrm{m})$ & $1051 \pm 250$ & $1030 \pm 578$ & Trivial & -293.825 to 333.748 \\
\hline HSE (no.) & $57 \pm 12$ & $56 \pm 32$ & Trivial & -16.649 to 17.560 \\
\hline RHSD (m/min) & $13.8 \pm 3.1$ & $13.4 \pm 7.5$ & Trivial & -3.6813 to 4.4742 \\
\hline $\mathrm{HS}(\%)$ & $11.4 \pm 23$ & $10.0 \pm 4.2$ & Small & -.9682 to 3.7529 \\
\hline
\end{tabular}

Difference mean value in high speed running (HSR), number of high speed efforts (HSE), relative high speed distance (RHSD) and percentage of time at high speed (HS \%); Trivial effect $\eta^{2}<0.01$; Small effect: $0.01<\eta^{2}<0.06$; Moderate effect $0.06<\eta^{2}<0.14$; Large effect: $\eta^{2}>0.14$. CI, confidence interval. $\ddagger$ Significant difference $(p<0.01)$ from division 1 team. ${ }^{*}$ Significant difference $(p<0.05)$ from division 1 team. 
TABLE 3

\begin{tabular}{lcccc}
\hline Variable & $\begin{array}{c}\text { Division } 1 \\
\text { Total } \\
(n=13 \text { games })\end{array}$ & $\begin{array}{c}\text { Division } 3 \\
\text { Total } \\
(n=12 \text { games })\end{array}$ & $\begin{array}{c}\text { Effect Size } \\
\text { Total Hand passes }\end{array}$ & $\begin{array}{c}\text { Difference } 95 \% \\
\text { CI }\end{array}$ \\
Total Kick passes & $71 \pm \pm 23$ & $167 \pm 36^{*}$ & Large & -60.44 to -10.87 \\
Shots Missed from Play & $9 \pm 3$ & $62 \pm 17$ & Medium & -2.07 to 21.53 \\
Shots Scored from Play & $10 \pm 4.1$ & $13 \pm 3^{*}$ & Large & -6.28 to -1.50 \\
Percentage of Possession & $49 \pm 3$ & $11 \pm 3$ & Small & -3.84 to 2.29 \\
Tackles in Defensive Third & $56 \pm 34$ & $34 \pm 10$ & Small & -4.09 to 2.19 \\
Tackles in Middle Third & $54 \pm 44$ & $36 \pm 11^{*}$ & Medium & -9.51 to 44.09 \\
Tackles in Attacking Third & $12 \pm 8$ & $10 \pm 4$ & Small & -3.30 to 7.32 \\
Total Tackles & $121 \pm 71$ & $80 \pm 17$ & Large & -2.65 to 84.29
\end{tabular}

Difference mean value; Small effect: $0.01<\eta^{2}<0.06$; Moderate effect $0.06<\eta^{2}<0.14$; Large effect: $\eta^{2}>0.14$. CI, confidence interval. * Significant difference $(\mathrm{p}<0.05)$ from Division 1 team 
FIGURE 1

2400

$\ddagger$

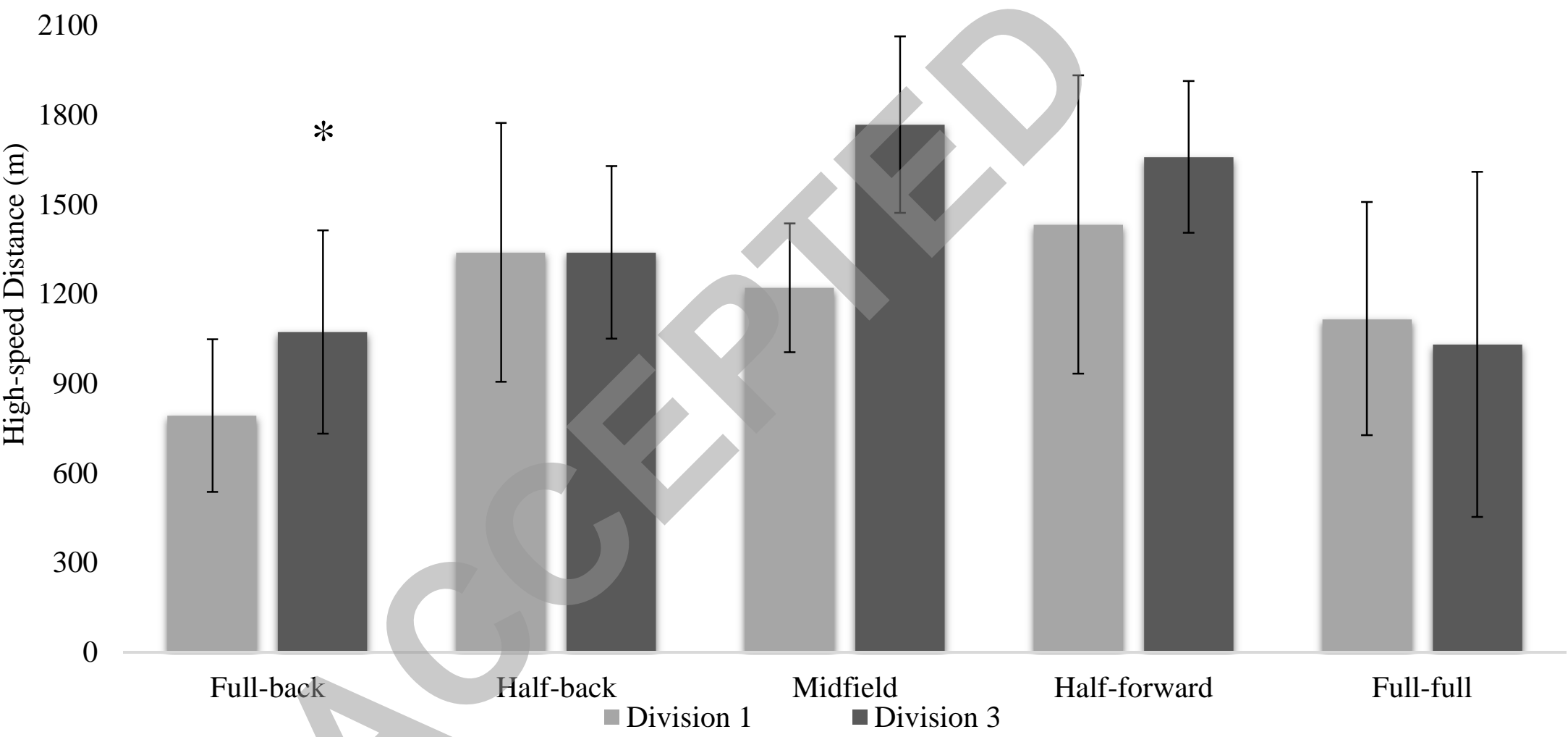

Difference means values significant difference set at 0.05 . $\ddagger$ Significantly different $(p<0.01)$ from Division 1 team

${ }^{*}$ Significant difference $(p<0.05)$ from Division 1 team. 